\title{
STABILIZATION OF WAVE SYSTEMS WITH INPUT DELAY IN THE BOUNDARY CONTROL*
}

\author{
Gen Qi Xu ${ }^{1}$, Siu Pang Yung ${ }^{2}$ And LeOng Kwan Li $^{3}$
}

\begin{abstract}
In the present paper, we consider a wave system that is fixed at one end and a boundary control input possessing a partial time delay of weight $(1-\mu)$ is applied over the other end. Using a simple boundary velocity feedback law, we show that the closed loop system generates a $C_{0}$ group of linear operators. After a spectral analysis, we show that the closed loop system is a Riesz one, that is, there is a sequence of eigenvectors and generalized eigenvectors that forms a Riesz basis for the state Hilbert space. Furthermore, we show that when the weight $\mu>\frac{1}{2}$, for any time delay, we can choose a suitable feedback gain so that the closed loop system is exponentially stable. When $\mu=\frac{1}{2}$, we show that the system is at most asymptotically stable. When $\mu<\frac{1}{2}$, the system is always unstable.
\end{abstract}

Mathematics Subject Classification. 34H05, 49J25, 49K25, 93D15.

Received July 1st, 2004. Revised May 10 and October 13, 2005.

\section{INTRODUCTION}

It is well known that time delay effects arise frequently in daily life practical problems. These hereditary effects are sometime unavoidable because they might turn a well-behave system into a wild one. A simple example can be found in Gumowski and Mira [1], where they demonstrated that the occurrence of delays could destroy the stability and cause periodic oscillations in a system governed by differential equation. Another examples from Datko [2,3] illustrated that an arbitrary small time delay in the control could destabilize a boundary feedback hyperbolic control system. On the other side, the inclusion of an appropriate time delay effect can sometime improve the performance of the system (e.g., see [4-8]). From the analytic point of view, a time delay system is in fact an infinite-dimensional system because the number of poles is usually infinite. So there could be infinitely many unstable poles in the system [2]. This makes the design of a stabilizing control a little harder that of the usual lumped parameter systems. There are many approaches that can be used. For example, via the characteristic roots of retarded and neutral functional differential equations [9], the Krasovskii-type approach [10,11], the Rasumikhim-type approach [9], and the control Lyapunov function approach [12].

Keywords and phrases. Wave equation, time delay, stabilization, Riesz basis.

* This research is supported by the Natural Science Foundation of China grant NSFC-60474017, by the Liu Hui Center for Applied Mathematics of the Nankai University 8 Tianjin University, by an Earmarked HKRGC grant 7053/03P and 7055/04P

1 Mathematics Department of Tianjin University, Tianjin, 300072, P.R. China; gqxu@tju.edu.cn

2 Mathematics Department of Hong Kong University, Hong Kong, P.R. China; spyung@hku.hk

3 Applied Mathematics Department of the Hong Kong Polytechnic University, Hong Kong, P.R. China; malblkli@polyu.edu.hk 
Recently, boundary feedbacks are used to design stabilizing controllers to overcome the negative effect of time delays (see $[13,14]$ and the references therein). In this article, we shall design a collocated boundary feedback controller to stabilize a wave system that has an input delay effect. Our model is similar to that of [2], but our approach is different which allow us to obtain a series of new properties for the system.

A usual wave system with boundary control is:

$$
\begin{cases}\ddot{w}(x, t)-w_{x x}(x, t)=0, & t>0 \quad x \in(0,1) \\ w(0, t)=0, & t>0 \\ w_{x}(1, t)=u(t), & t \geq 0 \\ w(x, 0)=w_{0}(x), \quad \dot{w}(x, 0)=w_{1}(x), & x \in(0,1) \\ y(t)=\dot{w}(1, t) & \end{cases}
$$

where $\dot{w}(x, t)$ denotes the derivative with respect to time $t, w_{x}(x, t)$ the derivative with respect to the spatial variable $x, u(t)$ is a control input, and $y(t)$ is an observation of the system.

If the control input $u(t)$ has no delay, we can use a simple feedback control law $u(t)=-k y(t)$, where $k>0$ is the feedback gain constant, to make the closed loop system dissipative and exponentially stable (e.g., see [15-17]). However, if the input has a small delay, the closed loop system becomes unstable as shown in [2].

So a nature question is how to stabilize system (1.1) when the input is allowed to have a time delay. To investigate this question, we split the control input into two parts: one has no delay and the other has a time delay, and a weighting of $\mu$ and $(1-\mu)$ are put respectively. More precisely, assume that $f(\theta)$, for $\theta \in(-\tau, 0)$, is a given function, and let $v(t)$ be an arbitrary function that satisfies

$$
v(t-\tau)=f(t-\tau), \quad \text { for } t \in(0, \tau)
$$

We suppose that the control input $u(t)$ is of the form

$$
u(t)=\mu v(t)+(1-\mu) v(t-\tau)
$$

where $\mu \in(0,1)$ is a parameter that denotes the weight of the time-delay effect: $\mu=1$ means no input delay, and $\mu=0$ means a full input delay. With this control input, system (1.1) become:

$$
\begin{cases}\ddot{w}(x, t)-w_{x x}(x, t)=0, & t>0 \quad x \in(0,1) \\ w(0, t)=0, & t>0 \\ w_{x}(1, t)=\mu v(t)+(1-\mu) v(t-\tau), & t \geq 0 \\ w(x, 0)=w_{0}(x), \quad \dot{w}(x, 0)=w_{1}(x), & x \in(0,1) \\ y(t)=\dot{w}(1, t) . & \end{cases}
$$

As usual, we adopt the simple feedback control law $v(t)=-k y(t)$ and result in the following closed loop system:

$$
\begin{cases}\ddot{w}(x, t)-w_{x x}(x, t)=0, & t>0 \quad x \in(0,1) \\ w(0, t)=0, & t>0 \\ w_{x}(1, t)=-k \mu \dot{w}(1, t)-k(1-\mu) \dot{w}(1, t-\tau), & t \geq 0 \\ w(x, 0)=w_{0}(x), \quad \dot{w}(x, 0)=w_{1}(x), & x \in(0,1) \\ \dot{w}(1, t-\tau)=f(t-\tau), \quad t \in(0, \tau) . & \end{cases}
$$


Setting $z(x, t)=\dot{w}(1, t-x \tau), x \in(0,1)$, equation (1.3) is equivalent to

$$
\begin{cases}\ddot{w}(x, t)-w_{x x}(x, t)=0, & t>0 \quad x \in(0,1), \\ \tau \dot{z}(x, t)+z_{x}(x, t)=0, & t>0, \quad x \in(0,1) \\ w(0, t)=0, \quad z(0, t)=\dot{w}(1, t) & t>0, \\ w_{x}(1, t)=-k \mu \dot{w}(1, t)-k(1-\mu) z(1, t), & t>0 \\ w(x, 0)=w_{0}(x), \quad \dot{w}(x, 0)=w_{1}(x), & x \in(0,1), \\ z(x, 0)=f(-x \tau), & x \in(0,1) .\end{cases}
$$

In this paper, we shall investigate the stability of system (1.4). We find that the stability would depend on the parameter $\mu$. More precisely, we find out that when $\mu>\frac{1}{2}$, system (1.4) is exponentially stable, but becomes unstable when $\mu<\frac{1}{2}$. When $\mu=\frac{1}{2}$, we find that if $\tau \in(0,1)$ is rational, then the the imaginary axis has at least one eigenvalue, and so the system is unstable. If $\tau \in(0,1)$ is irrational, we find that there are no eigenvalues on the imaginary axis, which in fact is an asymptote of the spectrum of the system, so the system is asymptotically stable.

Our main tool is a detail spectral analysis of system (1.4). We shall show that the spectrum determined growth condition is valid for system (1.4). Hence, various stabilities can be deduced from the information of the spectrum. To establish the spectrum determined growth condition, we show that system (1.4) is a Riesz system in that sense that: the multiplicities of all eigenvalues are uniformly bounded above, and there is a sequence of eigenvectors and generalized eigenvectors that forms a Riesz basis for the state Hilbert space.

The content of this paper is organized as follows. We shall formulate our problem in a suitable Hilbert space in Section 2 and show that the closed loop system generates a $C_{0}$ group of linear operators. In Section 3 , we carry out a spectral analysis and find out the locations and the multiplicities of the eigenvalues. The Riesz basis property of the generalized eigenfunctions will then be shown for system (1.4) and the spectrum determined growth condition will be deduced. Finally, in Section 4, we shall discuss the stability of system (1.4) according to different values of $\mu$.

\section{GRoup PROPERTY OF THE CLOSED LOOP SYSTEM}

In this section, we shall study some basic properties for system (1.4). We always assume that $\tau>0$ is fixed in the sequel. We first in the following Hilbert space $\mathcal{H}$ :

$$
\mathcal{H}=V^{1}[0,1] \times L^{2}[0,1] \times L^{2}[0,1],
$$

where $V^{k}(0,1)=\left\{f \in H^{k}(0,1) \mid f(0)=0\right\}, H^{k}(0,1)$ is the usual Sobolev space of order $k$. We equip $\mathcal{H}$ with the inner product

$$
\left(W_{1}, W_{2}\right)=\int_{0}^{1} u_{1}^{\prime}(x) \overline{u_{2}^{\prime}(x)} \mathrm{d} x+\int_{0}^{1} v_{1}(x) \overline{v_{2}(x)} \mathrm{d} x+\int_{0}^{1} \eta_{1}(x) \overline{\eta_{2}(x)} \mathrm{d} x, \quad \text { for } W_{j}=\left(u_{j}, v_{j}, \eta_{j}\right)^{T} \in \mathcal{H} .
$$

Define a linear operator $\mathcal{A}$ in $\mathcal{H}$ by

$$
\begin{gathered}
\mathcal{D}(\mathcal{A})=\left\{(u, v, \eta)^{T} \in V^{2}(0,1) \times V^{1}(0,1) \times H^{1}(0,1) \mid u^{\prime}(1)=-k \mu v(1)-k(1-\mu) \eta(1), v(1)=\eta(0)\right\}, \\
\mathcal{A}(u, v, \eta)^{T}=\left(v, u^{\prime \prime},-\tau^{-1} \eta^{\prime}\right)^{T}, \quad \forall(u, v, \eta)^{T} \in \mathcal{D}(\mathcal{A}) .
\end{gathered}
$$

Then, we can rewrite (1.4) as an evolutionary equation in $\mathcal{H}$ :

$$
\left\{\begin{array}{l}
\frac{\mathrm{d}}{\mathrm{d} t} W(t)=\mathcal{A} W(t), \quad t>0, \\
W(0)=W_{0}
\end{array}\right.
$$

where $W(t)=(w(x, t), \dot{w}(x, t), z(x, t))^{T}$, and $W_{0}=\left(w_{0}, w_{1}, f\right)^{T}$. Here come our first result. 
Theorem 2.1. Let $\mathcal{A}$ be defined by (2.1) and (2.2). Then for any $k, \mu \in \mathbb{R}, 0 \in \rho(\mathcal{A})$ and $\mathcal{A}^{-1}$ is compact on $\mathcal{H}$. Hence, $\sigma(\mathcal{A})$ is made up of isolated eigenvalues of finite multiplicity only.

Proof. Let $\mu, k \in \mathbb{R}$ be given. For any $F=(f, g, h) \in \mathcal{H}$, we consider the equation $\mathcal{A} W=F$, i.e.,

$$
v(x)=f(x), \quad u^{\prime \prime}(x)=g(x), \quad \eta^{\prime}(x)=-\tau h(x)
$$

and

$$
u(0)=v(0)=0, \quad \eta(0)=v(1), u^{\prime}(1)=-k \mu v(1)-k(1-\mu) \eta(1) .
$$

First, $v(x)=f(x) \in V^{1}(0,1)$. Second, if we solve the equation, we will get

$$
\eta(x)=\eta(0)-\tau \int_{0}^{x} h(s) \mathrm{d} s=f(1)-\tau \int_{0}^{x} h(s) \mathrm{d} s
$$

and

$$
u(x)=x u^{\prime}(1)-\int_{0}^{x} \mathrm{~d} s \int_{s}^{1} g(r) \mathrm{d} r=-k x f(1)+k(1-\mu) \tau x \int_{0}^{1} h(s) \mathrm{d} s-\int_{0}^{1} G(x, r) g(r) \mathrm{d} r,
$$

where $G(x, r)=\left\{\begin{array}{l}r, 0 \leq r \leq x, \\ x, x \leq r \leq 1 .\end{array}\right.$. Clearly such a vector $(u, v, \eta)$ belongs to $\mathcal{D}(\mathcal{A})$ and $\mathcal{A}^{-1}(f, g, h)=(u, v, \eta)$. Also, since $f \in V^{1}(0,1)$ and $u$ and $\eta$ are integrals of $g$ and $h$ respectively, so Sobolev's Embedding Theorem asserts that $\mathcal{A}^{-1}$ is a compact operator on $\mathcal{H}$.

To study the generation of a $C_{0}$ semigroup, we introduce a new inner product in $\mathcal{H}$ : for $W_{j}=\left(u_{j}, v_{j}, \eta_{j}\right) \in \mathcal{H}, j=1,2$, let

$$
\begin{aligned}
\left(W_{1}, W_{2}\right)_{1}= & \int_{0}^{1} \mathrm{e}^{\alpha x}\left[u_{1}^{\prime}(x)-v_{1}(x)\right] \overline{\left[u_{2}^{\prime}(x)-v_{2}(x)\right]} \mathrm{d} x+\int_{0}^{1} \mathrm{e}^{\beta x}\left[u_{1}^{\prime}(x)+v_{1}(x)\right] \overline{\left[u_{2}^{\prime}(x)+v_{2}(x)\right]} \mathrm{d} x \\
& +\tau \int_{0}^{1} \mathrm{e}^{\gamma x} \eta_{1}(x) \overline{\eta_{2}(x)} \mathrm{d} x
\end{aligned}
$$

It is easy to verify that $\left(W_{1}, W_{2}\right)_{1}$ is equivalent to the original inner product $\left(W_{1}, W_{2}\right)$. Under this new inner product, we have, for any real vector $W=(u, v, \eta) \in \mathcal{D}(\mathcal{A})$,

$$
\begin{aligned}
(\mathcal{A} W, W)_{1}= & \int_{0}^{1} \mathrm{e}^{\alpha x}\left[v^{\prime}(x)-u^{\prime \prime}(x)\right]\left[u^{\prime}(x)-v(x)\right] \mathrm{d} x+\int_{0}^{1} \mathrm{e}^{\beta x}\left[v^{\prime}(x)+u^{\prime \prime}(x)\right]\left[u^{\prime}(x)+v(x)\right] \mathrm{d} x \\
& -\int_{0}^{1} \mathrm{e}^{\gamma x} \eta^{\prime}(x) \eta(x) \mathrm{d} x \\
= & -\left.\frac{1}{2} \mathrm{e}^{\alpha x}\left|\left[u^{\prime}(x)-v(x)\right]\right|^{2}\right|_{0} ^{1}+\frac{\alpha}{2} \int_{0}^{1} \mathrm{e}^{\alpha x}\left|u^{\prime}(x)-v(x)\right|^{2} \mathrm{~d} x \\
& +\left.\frac{1}{2} \mathrm{e}^{\beta x}\left|\left[u^{\prime}(x)+v(x)\right]\right|^{2}\right|_{0} ^{1}-\frac{\beta}{2} \int_{0}^{1} \mathrm{e}^{\beta x}\left|u^{\prime}(x)+v(x)\right|^{2} \mathrm{~d} x \\
& -\left.\frac{1}{2} \mathrm{e}^{\gamma x}|\eta(x)|^{2}\right|_{0} ^{1}+\frac{\gamma}{2} \int_{0}^{1} \mathrm{e}^{\gamma x}|\eta(x)|^{2} \mathrm{~d} x \\
= & \frac{\alpha}{2} \int_{0}^{1} \mathrm{e}^{\alpha x}\left|u^{\prime}(x)-v(x)\right|^{2} \mathrm{~d} x-\frac{\beta}{2} \int_{0}^{1} \mathrm{e}^{\beta x}\left|u^{\prime}(x)+v(x)\right|^{2} \mathrm{~d} x+\frac{\gamma}{2} \int_{0}^{1} \mathrm{e}^{\gamma x}|\eta(x)|^{2} \mathrm{~d} x \\
& -\frac{1}{2} \mathrm{e}^{\alpha}\left|\left[u^{\prime}(1)-v(1)\right]\right|^{2}+\frac{1}{2} \mathrm{e}^{\beta}\left|\left[u^{\prime}(1)+v(1)\right]\right|^{2}-\frac{1}{2} \mathrm{e}^{\gamma}|\eta(1)|^{2}+\frac{1}{2}|\eta(0)|^{2} .
\end{aligned}
$$

Substituting the boundary conditions

$$
u^{\prime}(1)=-k \mu v(1)-k(1-\mu) \eta(1), \quad v(1)=\eta(0)
$$


lead to

$$
\begin{aligned}
(\mathcal{A} W, W)_{1}= & \frac{\alpha}{2} \int_{0}^{1} \mathrm{e}^{\alpha x}\left|u^{\prime}(x)-v(x)\right|^{2} \mathrm{~d} x-\frac{\beta}{2} \int_{0}^{1} \mathrm{e}^{\beta x}\left|u^{\prime}(x)+v(x)\right|^{2} \mathrm{~d} x+\frac{\gamma}{2} \int_{0}^{1} \mathrm{e}^{\gamma x}|\eta(x)|^{2} \mathrm{~d} x \\
& -\frac{1}{2} \mathrm{e}^{\alpha}|(k \mu+1) \eta(0)+k(1-\mu) \eta(1)|^{2}+\frac{1}{2} \mathrm{e}^{\beta}|(k \mu-1) \eta(0)+k(1-\mu) \eta(1)|^{2} \\
& -\frac{1}{2} \mathrm{e}^{\gamma}|\eta(1)|^{2}+\frac{1}{2}|\eta(0)|^{2} \\
= & \frac{\alpha}{2} \int_{0}^{1} \mathrm{e}^{\alpha x}\left|u^{\prime}(x)-v(x)\right|^{2} \mathrm{~d} x-\frac{\beta}{2} \int_{0}^{1} \mathrm{e}^{\beta x}\left|u^{\prime}(x)+v(x)\right|^{2} \mathrm{~d} x+\frac{\gamma}{2} \int_{0}^{1} \mathrm{e}^{\gamma x}|\eta(x)|^{2} \mathrm{~d} x \\
& -\frac{1}{2} G(\alpha, \beta, \gamma, k, \mu, \eta(0), \eta(1)),
\end{aligned}
$$

where

$$
\begin{aligned}
G(\alpha, \beta, \gamma, k, \mu, \eta(0), \eta(1))= & \eta^{2}(0)\left[\mathrm{e}^{\alpha}(k \mu+1)^{2}-\mathrm{e}^{\beta}(k \mu-1)^{2}-1\right] \\
& +\eta^{2}(1)\left[\mathrm{e}^{\alpha} k^{2}(1-\mu)^{2}-\mathrm{e}^{\beta} k^{2}(1-\mu)^{2}+\mathrm{e}^{\gamma}\right] \\
& +2 \eta(0) \eta(1)\left[(k \mu+1) k(1-\mu) \mathrm{e}^{\alpha}-(k \mu-1) k(1-\mu) \mathrm{e}^{\beta}\right] .
\end{aligned}
$$

Lemma 2.1. Let $k$ and $\mu$ be given with $k \mu+1 \neq 0$. Then we can choose real numbers $\alpha>0, \beta<0, \gamma>0$ such that

$$
4 \mathrm{e}^{\beta} k^{2}(1-\mu)^{2}+\mathrm{e}^{\beta-\alpha} k^{2}(1-\mu)^{2}+k^{2}(1-\mu)+\mathrm{e}^{\gamma+\beta-\alpha}(k \mu-1)^{2}+\mathrm{e}^{\gamma-\alpha}<\mathrm{e}^{\gamma}(k \mu+1)^{2} .
$$

Furthermore, under these choices, for any real $\eta(0), \eta(1)$, we have

$$
G(\alpha, \beta, \gamma, k, \mu, \eta(0), \eta(1))>0
$$

with $G$ defined above.

Proof. Let $k$ and $\mu$ be given with $k \mu+1 \neq 0$. We can choose $\alpha=\gamma>0$ large enough and $\beta<0$ such that

$$
\mathrm{e}^{\alpha}(k \mu+1)^{2}-\mathrm{e}^{\beta}(k \mu-1)^{2}-1>0
$$

and

$$
4 k^{2}(1-\mu)^{2}+k^{2}(1-\mu)^{2}+k^{2}(1-\mu)+(k \mu-1)^{2}+1<\mathrm{e}^{\gamma}(k \mu+1)^{2} .
$$

For such a choice of $\alpha, \beta$ and $\gamma$, we have

$$
\begin{aligned}
& {\left[(k \mu+1) k(1-\mu) \mathrm{e}^{\alpha}-(k \mu-1) k(1-\mu) \mathrm{e}^{\beta}\right]^{2} } \\
& -\left[\mathrm{e}^{\alpha}(k \mu+1)^{2}-\mathrm{e}^{\beta}(k \mu-1)^{2}-1\right] \times\left[\mathrm{e}^{\alpha} k^{2}(1-\mu)^{2}-\mathrm{e}^{\beta} k^{2}(1-\mu)^{2}+\mathrm{e}^{\gamma}\right] \\
= & 4 \mathrm{e}^{\beta} k^{2}(1-\mu)^{2}+\mathrm{e}^{\beta-\alpha} k^{2}(1-\mu)^{2}+k^{2}(1-\mu)+\mathrm{e}^{\gamma+\beta-\alpha}(k \mu-1)^{2}+\mathrm{e}^{\gamma-\alpha}-\mathrm{e}^{\gamma}(k \mu+1)^{2}<0 .
\end{aligned}
$$

So $G(\alpha, \beta, \gamma, k, \mu, \eta(0), \eta(1))>0$ and the proof is completed.

Lemma 2.2. Let $k$ and $\mu$ be given. We can then choose real numbers $\alpha<0, \beta>0, \gamma<0$ such that

$$
4 \mathrm{e}^{\beta} k^{2}(1-\mu)^{2}+\mathrm{e}^{\beta-\alpha} k^{2}(1-\mu)^{2}+k^{2}(1-\mu)+\mathrm{e}^{\gamma+\beta-\alpha}(k \mu-1)^{2}+\mathrm{e}^{\gamma-\alpha}>\mathrm{e}^{\gamma}(k \mu+1)^{2} .
$$

Also, under these choices, for any real $\eta(0), \eta(1)$, we have

$$
G(\alpha, \beta, \gamma, k, \mu, \eta(0), \eta(1))<0 .
$$

The proof is the same as that of Lemma 2.1. 
Theorem 2.2. Let $\mathcal{A}$ be defined by (2.1-2.2). Then $\mathcal{A}$ generates a $C_{0}$ group of linear operators on $\mathcal{H}$.

Proof. Let $\mathcal{A}$ be defined by (2.1-2.2). To show that $\mathcal{A}$ generates a $C_{0}$ semigroup on $\mathcal{H}$, we choose values for the parameters $\alpha, \beta$ and $\gamma$ as in Lemma 2.1. Then, for real vector $W=(u, v, \eta) \in \mathcal{D}(\mathcal{A})$, we know from Lemma 2.1 that

$$
(\mathcal{A} W, W)_{1}<\max \left\{\frac{\alpha}{2},-\frac{\beta}{2}, \frac{\gamma}{2 \tau}\right\}\|W\|_{1}^{2} .
$$

If we let $M=\max \left\{\frac{\alpha}{2},-\frac{\beta}{2}, \frac{\gamma}{2 \tau}\right\}$, then $\mathcal{A}-M I$ is dissipative, which together with Theorem 2.1 ensures that $\mathcal{A}$ generates a $C_{0}$ semigroup.

To prove that $-\mathcal{A}$ also generates a $C_{0}$ semigroup on $\mathcal{H}$, we can choose the values for parameters $\alpha, \beta$ and $\gamma$ as in Lemma 2.2. Then we have

$$
\begin{aligned}
-(\mathcal{A} W, W)_{1}= & -\frac{\alpha}{2} \int_{0}^{1} \mathrm{e}^{\alpha x}\left|u^{\prime}(x)-v(x)\right|^{2} \mathrm{~d} x+\frac{\beta}{2} \int_{0}^{1} \mathrm{e}^{\beta x}\left|u^{\prime}(x)+v(x)\right|^{2} \mathrm{~d} x-\frac{\gamma}{2} \int_{0}^{1} \mathrm{e}^{\gamma x}|\eta(x)|^{2} \mathrm{~d} x \\
& +\frac{1}{2} G(\alpha, \beta, \gamma, k, \mu, \eta(0), \eta(1)) \\
< & \max \left\{\frac{-\alpha}{2}, \frac{\beta}{2}, \frac{-\gamma}{2 \tau}\right\}\|W\|_{1}^{2} .
\end{aligned}
$$

Letting $M=\max \left\{\frac{-\alpha}{2}, \frac{\beta}{2}, \frac{-\gamma}{2 \tau}\right\}$, then $-\mathcal{A}-M I$ is dissipative and, hence, $-\mathcal{A}$ also generates a $C_{0}$ semigroup. Thus, $\mathcal{A}$ generates a $C_{0}$ group.

\section{SPECTRAL ANALYSIS}

From the previous section, we see that $\mathcal{A}$ generates a $C_{0}$ group. This implies that the spectrum of $\mathcal{A}$ lies in a vertical strip of the complex plane. In this section, we shall study the spectrum of $\mathcal{A}$ in more details. We begin with the characteristic equation.

Theorem 3.1. Let $\mathcal{A}$ be defined by (2.1-2.2) and let

$$
\Delta(\lambda)=\cosh \lambda+k \mu \sinh \lambda+k(1-\mu) \mathrm{e}^{-\lambda \tau} \sinh \lambda .
$$

Then $\sigma(\mathcal{A})=\sigma_{p}(\mathcal{A})=\{\lambda \in \mathbb{C} \mid \Delta(\lambda)=0\}$. For each $\lambda \in \sigma_{p}(\mathcal{A})$, the corresponding eigenspace has dimension one.

Proof. From Theorem 2.1, we know that $\sigma(\mathcal{A})=\sigma_{p}(\mathcal{A})$. For $\lambda \in \sigma(\mathcal{A})$, we consider the eigenvalue problem of $\mathcal{A}, \lambda W=\mathcal{A} W$, i.e.,

Solving these equations, we get

$$
\left\{\begin{array}{l}
\lambda u(x)=v(x) \\
\lambda v(x)=u^{\prime \prime}(x) \\
\lambda \eta(x)=-\frac{1}{\tau} \eta^{\prime}(x) \\
u(0)=v(0)=0 \\
u^{\prime}(1)=-k \mu v(1)-k(1-\mu) \eta(1) \\
v(1)=\eta(0)
\end{array}\right.
$$

$$
u(x)=a \sinh \lambda x, \quad v(x)=a \lambda \sinh \lambda x, \quad \eta(x)=\eta(0) \mathrm{e}^{-\lambda \tau x} .
$$

Substituting these expressions into the boundary conditions in (3.2), we get

$$
a[\lambda \cosh \lambda+k \mu \lambda \sinh \lambda]+k(1-\mu) \eta(0) \mathrm{e}^{\lambda \tau}=0, \quad a \lambda \sinh \lambda=\eta(0) .
$$


Since $0 \in \rho(\mathcal{A}),(3.3)$ has a non-trivial solution pair $(a, \eta(0))$ if and only if $\Delta(\lambda)=0$, i.e.,

$$
\Delta(\lambda):=\cosh \lambda+k \mu \sinh \lambda+k(1-\mu) \mathrm{e}^{-\lambda \tau} \sinh \lambda=0 .
$$

Therefore, $\sigma_{p}(\mathcal{A})=\{\lambda \in \mathbb{C} \mid \Delta(\lambda)=0\}$. Furthermore, an eigenvector corresponding to an eigenvalue $\lambda$ is given by

$$
W_{\lambda}=\left(\sinh \lambda x, \lambda \sinh \lambda x, \mathrm{e}^{-\lambda \tau x} \lambda \sinh \lambda\right) \in \mathcal{D}(\mathcal{A}) .
$$

So the dimension of the corresponding eigenspace is just one.

Since $\Delta(\lambda)$ is a function of real coefficients, so the following result is obvious.

Corollary 3.1. Let $\mathcal{A}$ be defined by (2.1-2.2). Then the spectrum of $\mathcal{A}$ distributes symmetrically with respect to the real axis, i.e., $\sigma(\mathcal{A})=\overline{\sigma(\mathcal{A})}$.

The following result describes the multiplicity and the separability of the eigenvalues of $\mathcal{A}$.

Theorem 3.2. Let $\Delta(\lambda)$ be defined by (3.1). Then the zeros of $\Delta(\lambda)$ are at most of degree two and separated from each others. If $\tau$ is irrational, then all zeros of $\Delta(\lambda)$ are simple and separated. If $\tau$ is rational, then all zeros of $\Delta(\lambda)$ lie on finitely many vertical lines in the complex plane.

Proof. Let $\Delta(\lambda)$ is defined by (3.1). Then from Theorem 2.1, we know that the zeros of $\Delta(\lambda)$ lie in a vertical strip parallel to the imaginary axis. To discuss the multiplicity of a zero of $\Delta(\lambda)$, let $\xi$ be a zero of $\Delta(\lambda)$. Then we have

Note that

$$
\Delta(\xi)=\cosh \xi+k \mu \sinh \xi+k(1-\mu) \mathrm{e}^{-\xi \tau} \sinh \xi=0 .
$$

and

$$
\Delta^{\prime}(\lambda)=\sinh \lambda+k \mu \cosh \lambda+k(1-\mu) \mathrm{e}^{-\lambda \tau} \cosh \lambda-k(1-\mu) \tau \mathrm{e}^{-\lambda \tau} \sinh \lambda
$$

$$
\begin{aligned}
\Delta^{\prime \prime}(\lambda) & =\cosh \lambda+k \mu \sinh \lambda+k(1-\mu) \mathrm{e}^{-\lambda \tau} \sinh \lambda-2 k(1-\mu) \tau \mathrm{e}^{-\lambda \tau} \cosh \lambda+k(1-\mu) \tau^{2} \mathrm{e}^{-\lambda \tau} \sinh \lambda \\
& =\Delta(\lambda)-2 k(1-\mu) \tau \mathrm{e}^{-\lambda \tau} \cosh \lambda+k(1-\mu) \tau^{2} \mathrm{e}^{-\lambda \tau} \sinh \lambda .
\end{aligned}
$$

We claim that $\Delta^{\prime \prime}(\xi) \neq 0$ whenever $\Delta(\xi)=\Delta^{\prime}(\xi)=0$. Note that $\Delta(\xi)=0$ implies $\sinh \xi \neq 0$. So we have

$$
\begin{aligned}
& \frac{\Delta(\xi)}{\sinh \xi}=\operatorname{coth} \xi+k \mu+k(1-\mu) \mathrm{e}^{-\xi \tau}, \\
& \frac{\Delta^{\prime}(\xi)}{\sinh \xi}=1+k \mu \operatorname{coth} \xi+k(1-\mu) \mathrm{e}^{-\xi \tau}[\operatorname{coth} \xi-\tau], \\
& \frac{\Delta^{\prime \prime}(\xi)}{\sinh \xi}=\frac{\Delta(\xi)}{\sinh \xi}-2 \tau k(1-\mu) \mathrm{e}^{-\xi \tau}\left[\operatorname{coth} \xi-\frac{\tau}{2}\right] .
\end{aligned}
$$

Hence, $\Delta(\xi)=0$ implies that

$$
-k(1-\mu) \mathrm{e}^{-\xi \tau}=\operatorname{coth} \xi+k \mu .
$$

Substitute this into the expression of $\frac{\Delta^{\prime}(\xi)}{\sinh \xi}$ leads to

$$
\frac{\Delta^{\prime}(\xi)}{\sinh \xi}=\left[\operatorname{coth} \xi-\frac{\tau+\sqrt{\tau^{2}+4(1+\tau k \mu)}}{2}\right]\left[\operatorname{coth} \xi-\frac{\tau-\sqrt{\tau^{2}+4(1+\tau k \mu)}}{2}\right] .
$$

So $\Delta^{\prime}(\xi)=0$ will give

$$
\operatorname{coth} \xi=\frac{\tau \pm \sqrt{\tau^{2}+4(1+\tau k \mu)}}{2}
$$


and therefore

$$
\Delta^{\prime \prime}(\xi)=-2 \tau k(1-\mu) \mathrm{e}^{-\xi \tau}\left[\operatorname{coth} \xi-\frac{\tau}{2}\right] \neq 0
$$

Thus, the zeros of $\Delta(\lambda)$ are at most of degree two.

Now suppose that $\xi$ is a zero of $\Delta(\lambda)$ of degree two. Then (3.5) and (3.7) must hold. Solving equation (3.7) gives

$$
\mathrm{e}^{2 \xi}= \begin{cases}\frac{\tau+\sqrt{\tau^{2}+4(1+\tau k \mu)}+2}{\tau+\sqrt{\tau^{2}+4(1+\tau k \mu)}-2}, & \text { if we take the }+ \text { sign } \\ \frac{\tau-\sqrt{\tau^{2}+4(1+\tau k \mu)}+2}{\tau-\sqrt{\tau^{2}+4(1+\tau k \mu)}-2}, & \text { if we take the }- \text { sign. }\end{cases}
$$

Substituting (3.7) into (3.5) yields

$$
\mathrm{e}^{\xi \tau}= \begin{cases}\frac{-2 k(1-\mu)}{\tau+\sqrt{\tau^{2}+4(1+\tau k \mu)}+2 k \mu}, & \text { if we take the }+ \text { sign } \\ \frac{-2 k(1-\mu)}{\tau-\sqrt{\tau^{2}+4(1+\tau k \mu)}+2 k \mu}, & \text { if we take the }-\operatorname{sign} .\end{cases}
$$

So either

or

$$
\left[\frac{2 k(1-\mu)}{\tau+\sqrt{\tau^{2}+4(1+\tau k \mu)}+2 k \mu}\right]^{2}=\left[\frac{\tau+\sqrt{\tau^{2}+4(1+\tau k \mu)}+2}{\tau+\sqrt{\tau^{2}+4(1+\tau k \mu)}-2}\right]^{\tau}
$$

$$
\left[\frac{2 k(1-\mu)}{\tau-\sqrt{\tau^{2}+4(1+\tau k \mu)}+2 k \mu}\right]^{2}=\left[\frac{\tau-\sqrt{\tau^{2}+4(1+\tau k \mu)}+2}{\tau-\sqrt{\tau^{2}+4(1+\tau k \mu)}-2}\right]^{\tau}
$$

or both must be true. Hence, if an eigenvalue makes one of these equalities false, then that eigenvalue must be simple.

Suppose that $\xi$ is a zero of $\Delta(\lambda)$ of degree two, and we let $\xi=x+i y$. Since the right sides of (3.8) and (3.9) are all real numbers (because $\tau, k, \mu$ are all nonnegative), so (3.8) implies sin $2 y=0$, and (3.9) implies $\sin y \tau=0$. Thus,

$$
y \tau=n \pi, \quad 2 y=m \pi,
$$

for some integer $n, m$. Hence, $\tau=\frac{2 n}{m}$ is rational. So if $\xi$ is a zero of degree two, then $\tau$ must be rational.

Suppose now that $\tau=\frac{2 n}{m}$ is rational with $n, m$ being some positive integers. If we set $\mathrm{e}^{\frac{\lambda}{m}}=z$, then $\Delta(\lambda)=0$ is equivalent to the following equation

$$
(1+k \mu) z^{2(m+n)}+(1-k \mu) z^{2 n}+k(1-\mu) z^{2 m}-k(1-\mu)=0
$$

We know that this equation has at most $2 m+2 n$ zeros. Let $z_{1}, z_{2}, \cdots, z_{r}$ be its zeros and set $z_{j}=\left|z_{j}\right| \mathrm{e}^{i \theta_{j}}$. Then

$$
\lambda_{j, \nu}=m \ln \left|z_{j}\right|+i m \theta_{j}+2 m \nu \pi i, \quad 1 \leq j \leq r, \quad \nu \in \mathbb{Z}
$$

are all the eigenvalues of $\mathcal{A}$ when $\tau$ is rational. Thus, the eigenvalues of $\mathcal{A}$ lies on finitely many vertical lines that contain those $\lambda_{j, \nu}$ listed in (3.11).

On the other hand, since $\lambda \in \sigma(\mathcal{A})$ implies

$$
0<\inf _{\lambda \in \sigma(\mathcal{A})}|\sinh \lambda| \leq \sup _{\lambda \in \sigma(\mathcal{A})}|\sinh \lambda|<\infty
$$

So

$$
\inf _{\lambda \in \sigma(\mathcal{A})}\left|\Delta^{\prime}(\lambda)\right|>0
$$


if and only if

$$
\inf _{\lambda \in \sigma(\mathcal{A})}\left|\left[\operatorname{coth} \lambda-\frac{\tau+\sqrt{\tau^{2}+4(1+\tau k \mu)}}{2}\right]\left[\operatorname{coth} \lambda-\frac{\tau-\sqrt{\tau^{2}+4(1+\tau k \mu)}}{2}\right]\right|>0 .
$$

Clearly, (3.12) holds when $\tau$ is irrational. So if $\tau$ is irrational, then $\inf _{\lambda \in \sigma(\mathcal{A})}\left|\Delta^{\prime}(\lambda)\right|>0$ and Theorem 3 of [16] implies the separability of the zeros in the sense that is, there is a $\delta>0$ so that

$$
\inf _{\xi \neq \lambda, \lambda, \xi \in \sigma(\mathcal{A})}|\lambda-\xi| \geq \delta>0 .
$$

This together with (3.11) conclude for the case when $\tau$ is rational that $\sigma(\mathcal{A})$ is separated.

In order to obtain the result of the completeness of eigenvectors and generalized eigenvectors of $\mathcal{A}$, we need the following lemma (see [16]).

Lemma 3.1. Let $\mathcal{B}$ be the generator of a $C_{0}$-semigroup in a Hilbert space $\mathcal{H}$. Assume that $\mathcal{B}$ is discrete and for $\lambda \in \rho\left(\mathcal{B}^{*}\right), R\left(\lambda, \mathcal{B}^{*}\right)$ is of the form

$$
R\left(\lambda, \mathcal{B}^{*}\right) Y=\frac{G(\lambda) Y}{F(\lambda)}, \quad \forall Y \in \mathcal{H}
$$

where for each $Y \in \mathcal{H}, G(\lambda) Y$ is an $\mathcal{H}$-valued entire function with order less than or equal to $\rho_{1}$ and $F(\lambda)$ is a scalar entire function of order $\rho_{2}$. Let $\rho=\max \left\{\rho_{1}, \rho_{2}\right\}<\infty$ and an integer $n$ so that $n-1 \leq \rho<n$. If there are $n+1$ rays $\gamma_{j}, j=0,1,2, \ldots, n$, on the complex plane $\arg \gamma_{0}=\frac{\pi}{2}<\arg \gamma_{1}<\arg \gamma_{2}<\cdots<\arg \gamma_{n}=\frac{3 \pi}{2}$ with $\arg \gamma_{j+1}-\arg \gamma_{j} \leq \frac{\pi}{n}, 0 \leq j \leq n-1$ such that $R\left(\lambda, \mathcal{B}^{*}\right) Y$ is bounded on each ray $\gamma_{j}, 0<j<n$ as $|\lambda| \longrightarrow \infty$ for any $Y \in \mathcal{H}$, then $\operatorname{Sp}(\mathcal{B})=\mathcal{H}$, where $\operatorname{Sp}(\mathcal{B})$ is defined as the closure of all linear combination of generalized eigenvectors of $\mathcal{B}$.

With the help of Lemma 3.1, we can prove the following result.

Theorem 3.3. Let $\mathcal{A}$ be defined by (2.1) and (2.2). Then the system of the generalized eigenvectors of $\mathcal{A}$ is complete in $\mathcal{H}$.

Proof. A direct calculation shows that the dual operator $\mathcal{A}^{*}$ of $\mathcal{A}$ has the form

$$
D\left(\mathcal{A}^{*}\right)=\left\{(f, g, h) \in V^{2} \times V^{1} \times H^{1} \mid, f^{\prime}(1)=k \mu g(1)+\tau^{-1} h(0), k(1-\mu) g(1)+\tau^{-1} h(1)=0\right\}
$$

and

$$
\mathcal{A}^{*}(f, g, h)=-\left(g(x), f^{\prime \prime}(x),-\tau^{-1} h^{\prime}(x)\right), \quad \forall(f, g, h) \in D\left(\mathcal{A}^{*}\right)
$$

For any $\lambda \in \rho\left(\mathcal{A}^{*}\right)$, and $Y=\left(y_{1}, y_{2}, y_{3}\right)^{T} \in \mathcal{H}$, we consider the resolvent problem of $\mathcal{A}^{*}$,

$$
\left(\lambda-\mathcal{A}^{*}\right) F=Y, \quad F=(f, g, h) \in D\left(\mathcal{A}^{*}\right),
$$

i.e.,

$$
\left\{\begin{array}{l}
\lambda f(x)+g(x)=y_{1}(x), \\
\lambda g(x)+f^{\prime \prime}(x)=y_{2}(x), \\
\tau \lambda h(x)-h^{\prime}(x)=\tau y_{3}(x), \\
f(0)=g(0)=0 \\
f^{\prime}(1)=k \mu g(1)-\tau^{-1} h(0) \\
k(1-\mu) g(1)+\tau^{-1} h(1)=0 .
\end{array}\right.
$$


Solving the differential equations yields

$$
\left\{\begin{array}{l}
f(x)=a \sinh \lambda x+\frac{1}{\lambda} \int_{0}^{x} \sinh \lambda(x-s)\left[y_{2}(s)-\lambda y_{1}(s)\right] \mathrm{d} s \\
g(x)=y_{1}(x)-\lambda f(x) \\
h(x)=h(0) \mathrm{e}^{\lambda \tau x}-\tau \int_{0}^{x} \mathrm{e}^{\lambda \tau(x-s)} y_{3}(s) \mathrm{d} s
\end{array}\right.
$$

Using the boundary conditions, we obtain the following algebraic equations:

$$
\left\{\begin{array}{l}
a[\lambda \cosh \lambda+k \mu \lambda \sinh \lambda]+\tau^{-1} h(0)=-G_{1}\left(y_{1}, y_{2}\right) \\
-a k(1-\mu) \lambda \sinh \lambda+\tau^{-1} h(0) \mathrm{e}^{\lambda \tau}=-G_{2}\left(y_{1}, y_{2}, y_{3}\right)
\end{array}\right.
$$

where

$$
\begin{aligned}
& G_{1}\left(y_{1}, y_{2}\right)=\int_{0}^{1} \cosh \lambda(1-s)\left[y_{2}(x)-\lambda y_{1}(s)\right] \mathrm{d} s+k \mu \int_{0}^{1} \sinh \lambda(1-s)\left[y_{2}(s)-\lambda y_{1}(s)\right] \mathrm{d} s-k \mu y_{1}(1) \\
& G_{2}\left(y_{1}, y_{2}, y_{3}\right)=k(1-\mu)\left[y_{1}(1)-\int_{0}^{1} \sinh \lambda(1-s)\left[y_{2}(s)-\lambda y_{1}(s)\right] \mathrm{d} s\right]-\int_{0}^{1} \mathrm{e}^{\lambda \tau(1-s)} y_{3}(s) \mathrm{d} s .
\end{aligned}
$$

Since $\lambda \in \rho\left(\mathcal{A}^{*}\right)$, so $\Delta(\lambda) \neq 0$, and

$$
\left\{\begin{array}{l}
a=\frac{\mathrm{e}^{-\lambda \tau} G_{2}-G_{1}}{\lambda \Delta(\lambda)} \\
h(0)=-\frac{\tau \mathrm{e}^{-\lambda \tau}}{\Delta(\lambda)}\left[k(1-\mu) \sinh \lambda G_{1}+(\cosh \lambda+k \mu \sinh \lambda) G_{2}\right] .
\end{array}\right.
$$

Thus, we obtain an expression for resolvent of $\mathcal{A}^{*}$ :

$$
F=(f, g, h)^{T}=R\left(\lambda, \mathcal{A}^{*}\right) Y=\left(\begin{array}{l}
a \sinh \lambda x+\frac{1}{\lambda} \int_{0}^{x} \sinh \lambda(s-s)\left[y_{2}(s)-\lambda y_{1}(s)\right] \mathrm{d} s \\
y_{1}(x)-a \lambda \sinh \lambda x-\int_{0}^{x} \sinh \lambda(x-s)\left[y_{2}(s)-\lambda y_{1}(s)\right] \mathrm{d} s \\
h(0) \mathrm{e}^{\lambda \tau x}-\tau \int_{0}^{x} \mathrm{e}^{\lambda \tau(x-s)} y_{3}(s) \mathrm{d} s
\end{array}\right)
$$

where $a, h(0)$ are given by (3.15). Note that $\Delta(\lambda) R\left(\lambda, \mathcal{A}^{*}\right) Y$ is an $\mathcal{H}$-valued entire function of finite exponential type, and if we denote it by $\mathcal{G}(\lambda) Y$, then we have

$$
R\left(\lambda, \mathcal{A}^{*}\right) Y=\frac{\mathcal{G}(\lambda) Y}{\Delta(\lambda)}, \quad \lambda \in \rho\left(\mathcal{A}^{*}\right)
$$

Since $\mathcal{A}$ generates a $C_{0}$ group, so on each of these rays $\gamma_{0}=-M+i \mathbb{R}_{+}, \gamma_{1}=-M-\mathbb{R}_{+}, \gamma_{2}=-M-i \mathbb{R}_{+}$for sufficient large positive real number $M, R\left(\lambda, \mathcal{A}^{*}\right) Y$ is uniformly bounded. Thus the conditions in Lemma 3.1 are fulfilled and the desired result follows.

The following result gives a criterion of Riesz basis sequence of the generalized eigenvectors of the generator of a $C_{0}$ semigroup, which is from [18].

Theorem 3.4. Let $\mathcal{H}$ be a separable Hilbert space, and $\mathcal{A}$ be the generator of $C_{0}$ semigroup $T(t)$. Suppose that 1) $\sigma(\mathcal{A})=\sigma_{1}(\mathcal{A}) \cup \sigma_{2}(\mathcal{A})$ and $\sigma_{2}(\mathcal{A})=\left\{\lambda_{k}\right\}_{k=1}^{\infty}$ consist of isolated eigenvalues of finite algebraic multiplicity;

2) $\sup _{k>1} m_{a}\left(\lambda_{k}\right)<\infty$, where $m_{a}\left(\lambda_{k}\right)=\operatorname{dim} E\left(\lambda_{k}, \mathcal{A}\right) \mathcal{H}$ and $E\left(\lambda_{k}, \mathcal{A}\right)$ is the Riesz projector associated with $\lambda_{k}$;

3) there is a constant $\alpha$ such that

$$
\sup \left\{\Re \lambda \mid \lambda \in \sigma_{1}(\mathcal{A})\right\} \leq \alpha \leq \inf \left\{\Re \lambda \mid \lambda \in \sigma_{2}(\mathcal{A})\right\}
$$


and

$$
\inf _{n \neq m}\left|\lambda_{n}-\lambda_{m}\right|>0
$$

Then the following assertions are true:

i) There exist two $T(t)$-invariant closed subspaces $\mathcal{H}_{1}$ and $\mathcal{H}_{2}$ with property that $\sigma\left(\left.\mathcal{A}\right|_{\mathcal{H}_{1}}\right)=\sigma_{1}(\mathcal{A})$ and $\sigma\left(\left.\mathcal{A}\right|_{\mathcal{H}_{2}}\right)=\sigma_{2}(\mathcal{A})$, and $\left\{E\left(\lambda_{k}, \mathcal{A}\right) \mathcal{H}_{2}\right\}_{k=1}^{\infty}$ forms a Riesz basis of subspaces for $\mathcal{H}_{2}$ (the definition of subspace Riesz basis can refer to [19]). Furthermore,

ii) If $\sup _{k \geq 1}\left\|E\left(\lambda_{k}, \mathcal{A}\right)\right\|<\infty$, then

$$
\mathcal{H}=\overline{\mathcal{H}_{1} \oplus \mathcal{H}_{2}}
$$

$$
\mathcal{D}(\mathcal{A}) \subset \mathcal{H}_{1} \oplus \mathcal{H}_{2} \subset \mathcal{H}
$$

iii) $\mathcal{H}$ has the decomposition

$$
\mathcal{H}=\mathcal{H}_{1} \oplus \mathcal{H}_{2}, \quad \text { (topological direct sum) }
$$

if and only if $\sup _{n \geq 1}\left\|\sum_{k=1}^{n} E\left(\lambda_{k}, \mathcal{A}\right)\right\|<\infty$.

Theorem 3.5. Let $\mathcal{A}$ be defined as (2.1-2.2). Then the generalized eigenvector system of $\mathcal{A}$ forms a Riesz basis in $\mathcal{H}$. Therefore system (2.3) satisfies the spectrum determined growth condition.

Proof. In order to obtain the desired result, we only need to verify the conditions in Theorem 3.4. If we take $\sigma_{1}(\mathcal{A})=\{-\infty\}$ and $\sigma_{2}(\mathcal{A})=\sigma(\mathcal{A})$, then Theorem 3.2 together with Theorem 3.1 shows that conditions 1) and 2) of Theorem 3.4 are fulfilled. Also, the group property and Theorem 3.2 ensure that condition 3) of Theorem 3.4 is satisfied. So Theorem 3.4 says that there exist invariant subspaces $\mathcal{H}_{1}$ and $\mathcal{H}_{2}$ such that $\overline{\mathcal{H}_{1}+\mathcal{H}_{2}}=\mathcal{H}$. Furthermore, there is a sequence of (generalized) eigenvectors of $\mathcal{A}$ that forms a Riesz basis of subspace for $\mathcal{H}_{2}$. Since Theorem 3.3 also asserts that $\mathcal{H}_{2}=\mathcal{H}$, so this sequence of generalized eigenvectors of $\mathcal{A}$ forms a Riesz basis for $\mathcal{H}$. Finally, the spectrum determined growth condition follows directly from this Riesz basis property.

\section{Exponential Stability of THE ClOSED LOOP SYSTEM}

In this section we shall investigate the stability of the controlled system (2.3). Note that there are two parameters $k$ and $\mu$ in this system. Our concern is that for given delay $\tau$, whether one can find a feedback gain constant $k$ which depends on $\mu$ such that the corresponding closed loop system is stable. We shall see that the system is exponentially stable when $\mu>1 / 2$, and unstable when $\mu<1 / 2$. When $\mu=1 / 2$, the system will be unstable when $\tau$ lies in some countable set but asymptotically stable when $\tau$ does not lie in that countable set.

To begin, we introduce a new inner product in $\mathcal{H}$ by

$$
\left(W_{1}, W_{2}\right)_{2}=\int_{0}^{1} u_{1}^{\prime}(x) \overline{u_{2}^{\prime}(x)} \mathrm{d} x+\int_{0}^{1} v_{1}(x) \overline{v_{2}(x)} \mathrm{d} x+\xi \tau \int_{0}^{1} \eta_{1}(x) \overline{\eta_{2}(x)} \mathrm{d} x, \quad \forall W_{j}=\left(u_{j}, v_{j}, \eta_{j}\right) \in \mathcal{H},
$$

where $\xi$ is positive parameter. Easy to see that this inner product is equivalent to $\left(W_{1}, W_{2}\right)$. Under this new inner product, for any $W \in \mathcal{D}(\mathcal{A})$, we have

$2 \operatorname{Re}(\mathcal{A} W, W)_{2}=(\mathcal{A} W, W)_{2}+(W, \mathcal{A} W)_{2}$

$$
\begin{aligned}
&=-[(2 k \mu-\xi) \eta(0) \overline{\eta(0)}+k(1-\mu) {[\eta(0) \overline{\eta(1)}+\eta(1) \overline{\eta(0)}]+\xi \eta(1) \overline{\eta(1)}] } \\
&=-[\eta(0) \eta(1)]\left[\begin{array}{cc}
2 k \mu-\xi & k(1-\mu) \\
k(1-\mu) & \xi
\end{array}\right]\left[\frac{\overline{\eta(0)}}{\eta(1)}\right] .
\end{aligned}
$$


Setting

$$
B=\left[\begin{array}{cc}
2 k \mu-\xi & k(1-\mu) \\
k(1-\mu) & \xi
\end{array}\right]
$$

we immediately obtain the following assertion.

Proposition 4.1. Let $B$ be defined by (4.2). Then the eigenvalues of $B$ are given by

$$
\lambda_{1,2}=k \mu \pm \sqrt{k^{2} \mu^{2}+k^{2}(1-\mu)^{2}-2 k \mu \xi+\xi^{2}} .
$$

Therefore,(4.1) is non-positive if and only if

$$
g(k, \mu, \xi):=k^{2}(1-\mu)^{2}-2 k \mu \xi+\xi^{2} \leq 0
$$

Proposition 4.2. Let $g(k, \mu, \xi)$ be given by (4.4) with domain $(0, \infty) \times(0,1) \times(0, \infty)$. Then the following statements are true.

1) The function $g$ decreases strictly with respect to $\mu \in(0,1)$ and hence there exists a unique $\mu_{0} \in(0,1)$ such that $g(k, \mu, \xi)>0, \forall \mu \in\left(0, \mu_{0}\right)$ and $g(k, \mu, \xi)<0, \forall \mu \in\left(\mu_{0}, 1\right)$ with condition $\xi \leq 2 k$.

2) The zero point $\mu_{0}$ of $g(k, \mu, \xi)$ is given by

$$
\mu_{0}(k, \xi):=\frac{k+\xi-\sqrt{2 k \xi}}{k}
$$

3) Also, $\mu_{0}(k, \xi)$ on the domain $D=\{(k, \xi) \mid k \in(0, \infty), 0<\xi \leq 2 k\}$ has an interior point and its minimum value is $\frac{1}{2}$.

Proof. Let $g(k, \mu, \xi)$ be defined by (4.4). Then, since $\mu \in(0,1), k>0$ and $\xi>0$, so

$$
\frac{\partial g}{\partial \mu}=-2 k(1-\mu)-2 k \xi<0
$$

and hence the first assertion is true. To solve for the zero of $g(k, \mu, \xi)$ in $\mu \in(0,1)$, we solve the equation

$$
k^{2}(1-\mu)^{2}-2 k \mu \xi+\xi^{2}=0
$$

directly to yields

$$
\mu_{0}(k, \xi):=\frac{k+\xi-\sqrt{2 k \xi}}{k}, \quad 0<\xi \leq 2 k .
$$

Note that the function $\mu_{0}(k, \xi)$ on the domain

$$
D=\{(k, \xi) \mid k \in(0, \infty), 0<\xi \leq 2 k\}
$$

is bounded, and $\left.\mu_{0}(k, \xi)\right|_{\partial D}=1$ with $\mu_{0}(0,0)$ being defined to be 1 . So $\mu_{0}(k, \xi)$ takes its minimum value in the interior of $D$. Since

$$
\frac{\partial \mu_{0}}{\partial k}=-\xi k^{-2}+\frac{\sqrt{2 \xi}}{2} k^{-3 / 2}, \quad \frac{\partial \mu_{0}}{\partial \xi}=\frac{2 \sqrt{\xi}-\sqrt{2 k}}{2 k \sqrt{\xi}}
$$

so $\frac{\partial \mu_{0}}{\partial k}=\frac{\partial \mu_{0}}{\partial \xi}=0$ implies that $k=2 \xi$. Thus

$$
\min _{D} \mu_{0}(k, \xi)=\mu_{0}(2 \xi, \xi)=\frac{1}{2}
$$


Theorem 4.1. Let $\tau>0$ and $\mu \neq 0$ be fixed and let $\mathcal{A}$ be defined as (2.1-2.2). Then the following assertions hold.

1) When $\mu>\frac{1}{2}$, we can choose $k>0$ such that $\mathcal{A}$ is dissipative under the inner product $(W, Z)_{2}$, and there exists no eigenvalue on the imaginary axis. Hence, the system is asymptotically stable in this case.

2) If $\mu=\frac{1}{2}$ and $\tau \in J$ with

$$
J:=\left\{\frac{2(2 m+1)}{2 n+1}, \mid n, m \in \mathbb{N}\right\} \cap(0,1],
$$

then there exists at least one eigenvalue on the imaginary axis - so the system is unstable. If $\mu=\frac{1}{2}$ but $\tau \in(0,1) \backslash J$, then the system is asymptotically stable.

3) When $\mu<\frac{1}{2}$, then for any $\tau>0$, there exists an eigenvalue $\lambda$ of $\mathcal{A}$ with $\operatorname{Re} \lambda>0$. So, in this case, the system always is unstable.

Proof. Let $\tau$ and $\xi$ be both fixed and assume $\mu \geq \frac{1}{2}$. We want to choose a positive number $k$ so that

$$
g(k, \mu, \xi)=k^{2}(1-\mu)^{2}-2 k \mu \xi+\xi^{2} \leq 0,
$$

and we can have

1) If $\mu>\frac{1}{2}$, we can take

$$
2 \operatorname{Re}(\mathcal{A} W, W)_{2} \leq 0
$$

$$
k \in\left[\frac{\xi(\mu-\sqrt{2 \mu-1})}{(1-\mu)^{2}}, \frac{\xi(\mu+\sqrt{2 \mu-1})}{(1-\mu)^{2}}\right]
$$

so that $g(k, \mu, \xi) \leq 0$. In particular, we can choose $k$ so that $g(k, \mu, \xi)<0$ to make $B$ a positive definite matrix. Under this choice, we have

$$
\operatorname{Re}(A W, W)_{2}=-[\eta(0), \eta(1)] B \overline{[\eta(0), \eta(1)]}^{\tau}<0, \quad \forall W \in \mathcal{D}(\mathcal{A})
$$

Also, if $\lambda \in i \mathbb{R}$ is an eigenvalue with eigenvector $W_{\lambda}$, then $\operatorname{Re}(A W, W)_{2}=0$ which is impossible. Thus, there is no eigenvalue on the imaginary axis and the system is asymptotically stable.

2) Assume that $\mu=\frac{1}{2}$, we can take $k=2 \xi$ to have $g(2 \xi, 1 / 2, \xi)=0$ and so

$$
2 \operatorname{Re}(\mathcal{A} W, W)_{2} \leq 0
$$

Hence, $\mathcal{A}$ is dissipative.

To see whether there is an eigenvalue on the imaginary axis, let $\lambda \in i \mathbb{R}$ be an eigenvalue of $\mathcal{A}$ and $W_{\lambda}$ be the corresponding eigenvector. Then $\operatorname{Re}\left(\mathcal{A} W_{\lambda}, W_{\lambda}\right)_{2}=0$ which implies that $\eta(0)+\eta(1)=0$. From (3.4), we see that $W_{\lambda}$ is of the form

$$
W=(u, v, \eta)=\left(\sinh \lambda x, \lambda \sinh \lambda x, \mathrm{e}^{\lambda \tau x} \lambda \sinh \lambda\right) .
$$

So we must have

$$
\left\{\begin{array}{l}
\Delta(\lambda)=\cosh \lambda+k \mu \sinh \lambda+k(1-\mu) \mathrm{e}^{-\lambda \tau} \sinh \lambda=0, \quad \text { with } \mu=\frac{1}{2}, \\
1+\mathrm{e}^{-\lambda \tau}=0
\end{array}\right.
$$

which is just

$$
\mathrm{e}^{2 \lambda}=-1, \quad \text { and } \quad \mathrm{e}^{\lambda \tau}=-1 .
$$

Equation (4.7) would have at least one solution when $\tau \in J$ with

$$
J:=\left\{\frac{2(2 m+1)}{2 n+1}, \mid n, m \in \mathbb{N}\right\} \cap(0,1)
$$


Therefore, there is at least one eigenvalue of $\mathcal{A}$ on the imaginary axis. So the system is unstable. If $\tau \in(0,1) \backslash J$, then equation (4.7) has no solution. So there is no eigenvalue on the imaginary axis. Therefore, the system is asymptotically stable.

3) Now let $\mu<\frac{1}{2}$ and $k>0$. If $\tau=\frac{2(2 m+1)}{2 n+1}, n, m \in \mathbb{N}$, we set

$$
\lambda=\eta+i\left(n+\frac{1}{2}\right) \pi+i 2 r(2 n+1) \pi, \quad r \in \mathbb{Z},
$$

with some parameter $\eta$. Then

$$
\begin{array}{cl}
2 \lambda & =2 \eta+i(2 n+1) \pi+i 4 r(2 n+1) \pi, \quad r \in \mathbb{Z}, \\
\lambda \tau & =\eta \tau+i(2 m+1) \pi+i 4 r(2 m+1) \pi, \quad r \in \mathbb{Z},
\end{array}
$$

and hence

$$
\begin{aligned}
2 \mathrm{e}^{-\lambda} \Delta(\lambda) & =2 \mathrm{e}^{-\lambda} \cosh \lambda+2\left[k \mu+k(1-\mu) \mathrm{e}^{-\lambda \tau}\right] \mathrm{e}^{-\lambda} \sinh \lambda \\
& =\left[1+\mathrm{e}^{-2 \lambda}\right]+\left[k \mu+k(1-\mu) \mathrm{e}^{-\lambda \tau}\right]\left[1-\mathrm{e}^{-2 \lambda}\right] \\
& =\left[1-\mathrm{e}^{-2 \eta}\right]+\left[k \mu-k(1-\mu) \mathrm{e}^{-\eta \tau}\right]\left[1+\mathrm{e}^{-2 \eta}\right]:=\Delta_{0}(\eta) .
\end{aligned}
$$

Since

$$
\Delta_{0}(0)=2 k[2 \mu-1]<0, \quad \lim _{\eta \rightarrow+\infty} \Delta_{0}(\eta)=1+k \mu>0,
$$

so there is an $\eta>0$ such that $\Delta_{0}(\eta)=0$. Thus for this $\eta$, the complex numbers $\lambda$ given by (4.8) are eigenvalues of $\mathcal{A}$.

Now let $\tau>0$ be any positive real number, then we can choose a sequence of rationals, $\tau_{n, m}:=\frac{2(2 m+1)}{2 n+1}$, such that $\lim _{n, m \rightarrow \infty} \tau_{n, m}=\tau$. Denoting $\tau=\tau_{n, m}+\varepsilon_{n, m}$, we compare

$$
\Delta_{\tau}(\lambda)=\lambda^{-1}\left\{\cosh \lambda+\left[k \mu+k(1-\mu) \mathrm{e}^{-\lambda \tau}\right] \sinh \lambda\right\}
$$

with

$$
\Delta_{\tau_{n, m}}(\lambda)=\lambda^{-1}\left\{\cosh \lambda+\left[k \mu+k(1-\mu) \mathrm{e}^{-\lambda \tau_{n, m}}\right] \sinh \lambda\right\} .
$$

Suppose that $\lambda_{n, m}$ is a zero for $\Delta_{\tau_{n, m}}(\lambda)$ with positive real part. Then for $\left|\lambda-\lambda_{n, m}\right|=\frac{1}{2} \operatorname{Re} \lambda_{n, m}$, we have

$$
\left|\Delta_{\tau}(\lambda)-\Delta_{\tau_{n, m}}(\lambda)\right|=k(1-\mu)\left|\lambda^{-1}\left[\mathrm{e}^{-\lambda \tau}-\mathrm{e}^{-\lambda \tau_{n, m}}\right] \sinh \lambda\right| \leq k(1-\mu) \mathrm{e}^{-\Re \lambda \tau}\left|\tau-\tau_{n, m}\right| \cosh \operatorname{Re} \lambda<\left|\Delta_{\tau_{n, m}}(\lambda)\right|
$$

So Rouché's Theorem (see [20]) says that $\Delta_{\tau}(\lambda)$ and $\Delta_{\tau_{n, m}}(\lambda)$ have the same number of zeros in $\left|\lambda-\lambda_{n, m}\right|<$ $\frac{1}{2} \Re \lambda_{n, m}$. Since $\Delta_{\tau}(\lambda)$ has at least one zero with positive real part, so the same is true for $\Delta(\lambda)$ and hence the system is unstable.

For the case that $\mu>\frac{1}{2}$, we seek to improve the stability to exponential stability. For that, we need to prove that the imaginary axis is not an asymptote of the spectrum of $\mathcal{A}$. When $\tau>0$ is rational, Theorem 3.2 already reveals that the eigenvalues of $\mathcal{A}$ lie on finitely many vertical lines in the open left half complex plane, and so exponential stability is valid in this case. We now show that exponential stability is also true when $\tau$ is irrational by showing that the imaginary axis is not an asymptote of $\sigma(\mathcal{A})$.

Theorem 4.2. Let $\mathcal{A}$ be defined by (2.1-2.2). Then the system (2.3) is exponentially stable for a suitable chosen $k$ provided $\mu>\frac{1}{2}$. 
Proof. As we have discussed, the cases that $\tau$ is rational are fine. Let $\tau>0$ be an irrational number and $\mu>\frac{1}{2}$. Then we can choose $k>0$ such that $g(k, \mu, \xi)<0$, and hence $B$ is a positive definite matrix. So there is a positive constant $c_{1}$ such that

$$
[x, y] B[x, y]^{\tau} \geq c_{1}\left(|x|^{2}+|y|^{2}\right), \quad \forall x, y \in \mathbb{R}
$$

For such a choice, we have

$$
2 \operatorname{Re}(\mathcal{A} W, W)_{2}=-[\eta(0), \eta(1)] B[\eta(0), \eta(1)]^{\tau}<-c_{1}\left(|\eta(0)|^{2}+|\eta(1)|^{2}\right), \quad \forall W \in \mathcal{D}(\mathcal{A})
$$

Suppose that there exist $\lambda_{m} \in \sigma(\mathcal{A})$ such that $\operatorname{Re} \lambda_{m} \rightarrow 0$ as $m \rightarrow \infty$. Then

$$
W_{\lambda_{m}}=\left(\sinh \lambda_{m} x, \lambda_{m} \sinh \lambda_{m}, \mathrm{e}^{-\lambda_{m} \tau x} \lambda_{m} \sinh \lambda_{m}\right)
$$

would be an eigenvector corresponding to $\lambda_{m}$, and

$$
2 \operatorname{Re}\left(\mathcal{A} W_{\lambda_{m}}, W_{\lambda_{m}}\right)_{2}=2 \operatorname{Re} \lambda_{m}|| W_{\lambda_{m}} \|_{2}^{2}<-c_{1}\left[\left|\lambda_{m} \sinh \lambda_{m}\right|^{2}+\left|\mathrm{e}^{-\lambda_{m} \tau} \lambda_{m} \sinh \lambda_{m}\right|^{2}\right] .
$$

Observe that

$$
\begin{aligned}
\left\|W_{\lambda_{m}}\right\|^{2} & =\int_{0}^{1}\left|\lambda_{m} \cosh \lambda_{m} x\right|^{2}+\int_{0}^{1}\left|\lambda_{m} \sinh \lambda_{m} x\right|^{2}+\tau \xi \int_{0}^{1} \mathrm{e}^{-2 \operatorname{Re} \lambda_{m} \tau x} \mathrm{~d} x\left|\lambda_{m} \sinh \lambda\right|^{2} \\
& =\left|\lambda_{m}\right|^{2} \int_{0}^{1}\left[\left|\cosh \lambda_{m} x\right|^{2}+\left|\sinh \lambda_{m} x\right|^{2}\right] \mathrm{d} x+\tau \xi\left|\lambda_{m} \sinh \lambda_{m}\right|^{2} \int_{0}^{1} \mathrm{e}^{-2 \operatorname{Re} \lambda_{m} \tau x} \mathrm{~d} x \\
& =\left|\lambda_{m}\right|^{2} \frac{\sinh 2 \operatorname{Re} \lambda_{m}}{2 \operatorname{Re} \lambda_{m}}+\left|\lambda_{m} \sinh \lambda_{m}\right|^{2} \frac{1-\mathrm{e}^{-2 \operatorname{Re} \lambda_{m} \tau}}{2 \operatorname{Re} \lambda_{m}} \xi
\end{aligned}
$$

and

$$
\frac{\left\|W_{\lambda_{m}}\right\|^{2}}{\left|\lambda_{m}\right|^{2}}=\frac{\sinh 2 \operatorname{Re} \lambda_{m}}{2 \operatorname{Re} \lambda_{m}}+\left|\sinh \lambda_{m}\right|^{2} \frac{1-\mathrm{e}^{-2 \operatorname{Re} \lambda_{m} \tau}}{2 \operatorname{Re} \lambda_{m}} \xi=1+\xi \tau\left|\sinh \lambda_{m}\right|^{2}+0\left(\operatorname{Re} \lambda_{m}\right) .
$$

Thus

$$
\operatorname{Re} \lambda_{m}<-c_{1} \frac{\left|\sinh \lambda_{m}\right|^{2}}{1+\left|\sinh \lambda_{m}\right|^{2}+o\left(\operatorname{Re} \lambda_{m}\right)}\left[1+\mathrm{e}^{-2 \operatorname{Re} \lambda_{m} \tau}\right]
$$

Since $\sinh \lambda_{m} \nrightarrow 0$ as $m \rightarrow \infty$ for $\lambda_{m} \in \sigma(\mathcal{A})$, so the left hand of (4.9) converges to zero by assumption. However, the right hand of (4.9) cannot converge to zero when $\operatorname{Re} \lambda_{m} \rightarrow 0$. This is a contradiction so such a sequence $\lambda_{m} \in \sigma(\mathcal{A})$ with $\operatorname{Re} \lambda_{m} \rightarrow 0$ cannot exist. Thus the imaginary axis is not an asymptote for $\sigma(\mathcal{A})$. Hence system (2.3) is exponentially stable.

Remark 4.1. When $\mu=\frac{1}{2}$, for any $\tau \in(0,1) \backslash J$, Theorem 4.1 says that the system is asymptotically stable. One can prove that the imaginary axis is an asymptote of $\sigma(\mathcal{A})$. So, in this case, the system is not exponentially stable.

In this paper, we mainly discuss the boundary stabilization of the wave system. What would happen if the controller is in the equation instead of on the boundary? We shall investigate this stabilization problem in our forthcoming paper.

Acknowledgements. The authors would like to thank the referees for their useful and helpful comments and suggestions. 


\section{REFERENCES}

[1] I. Gumowski and C. Mira, Optimization in Control Theory and Practice. Cambridge University Press, Cambridge (1968).

[2] R. Datko, J. Lagness and M.P. Poilis, An example on the effect of time delays in boundary feedback stabilization of wave equations. SIAM J. Control Optim. 24 (1986) 152-156.

[3] R. Datko, Not all feedback stabilized hyperbolic systems are robust with respect to small time delay in their feedbacks. SIAM J. Control Optim. 26 (1988) 697-713.

[4] I.H. Suh and Z. Bien, Use of time delay action in the controller design. IEEE Trans. Automat. Control 25 (1980) 600-603.

[5] W.H. Kwon, G.W. Lee and S.W. Kim, Performance improvement, using time delays in multi-variable controller design. INT J. Control 52 (1990) 1455-1473.

[6] G. Abdallah, P. Dorato, J. Benitez-Read and R. Byrne, Delayed positive feedback can stabilize oscillatory systems, in ACC' 93 (American control conference), San Francisco (1993) 3106-3107.

[7] N. Jalili and N. Olgac, Optimum delayed feedback vibration absorber for MDOF mechanical structure, in 37th IEEE CDC'98 (Conference on decision and control), Tampa, FL, December (1998) 4734-4739.

[8] W. Aernouts, D. Roose and R. Sepulchre, Delayed control of a Moore-Greitzer axial compressor model. Intern. J. Bifurcation Chaos 10 (2000) 115-1164.

[9] J.K. Hale and S.M. Verduyn-Lunel, Strong stabilization of neutral functional differential equations. IMA J. Math. Control Inform. 19 (2002) 5-24.

[10] J.K. Hale and S.M. Verduyn-Lunel, Introduction to functional differential equations, in Applied Mathematical Sciences, New York, Springer 99 (1993).

[11] S.I. Niculescu and R. Lozano, On the passivity of linear delay systems. IEEE Trans. Automat. Control 46 (2001) $460-464$.

[12] P. Borne, M. Dambrine, W. Perruquetti and J.P. Richard, Vector Lyapunov functions: nonlinear, time-varying, ordinary and functional differential equations. Stability and control: theory, methods and applications 13, Taylor and Francis, London (2003) 49-73.

[13] Ö. Mörgul, On the stabilization and stability robustness against small delays of some damped wave equation. IEEE Trans. Automat. Control 40 (1995) 1626-1630.

[14] Ö. Mörgul, Stabilization and disturbance rejection for the wave equation. IEEE Trans. Automat. Control 43 (1998) 89-95.

[15] J.-L. Lions, Exact controllability, stabilization and perturbations for distributed parameter system. SIAM Rev. 30 (1988) 1-68.

[16] G.Q. Xu and B.Z. Guo, Riesz basis property of evolution equations in Hilbert spaces and application to a coupled string equation. SIAM J. Control Optim. 42 (2003) 966-984.

[17] M.A. Shubov, The Riesz basis property of the system of root vectors for the equation of a nonhomogeneous damped string: transformation operators method. Methods Appl. Anal. 6 (1999) 571-591.

[18] G.Q. Xu and S.P. Yung, The expansion of semigroup and a criterion of Riesz basis. J. Differ. Equ. 210 (2005) 1-24.

[19] I.C. Gohberg and M.G. Krein, Introduction to the Theory of Linear Nonselfadjoint Operators. AMS Transl. Math. Monographs 18 (1969).

[20] Lars V. Ahlfors, Complex Analysis. McGraw-Hill. 